\title{
Multiple-relaxation-time lattice Boltzmann simulation for flow, mass transfer and adsorption in porous media
}

\author{
Qiang Ma ${ }^{1,2,3}$, Zhenqian Chen, ${ }^{3, *}$ and Hao Liu ${ }^{2, \#}$ \\ ${ }^{1}$ Institute for Energy Research, Jiangsu University, 301 Xuefu Road, Zhenjiang 212013, P. R. \\ China \\ ${ }^{2}$ Faculty of Engineering,University of Nottingham,University Park, Nottingham NG72RD,UK \\ ${ }^{3}$ Jiangsu Provincial Key Laboratory of Solar Energy Science and Technology, School of Energy \\ and Environment, Nanjing 210096, P. R. China
}

In this paper, to predict the dynamics behaviors of flow and mass transfer with adsorption phenomena in porous media at the representative elementary volume (REV) scale, a multiple-relaxation-time (MRT) lattice Boltzmann (LB) model for the convection-diffusion equation is developed to solve the transfer problem with an unsteady source term in porous media. Utilizing the Chapman-Enskog analysis, the modified MRT-LB model can recover the macroscopic governing equations at the REV scale. The coupled MRT-LB model for momentum and mass transfer is validated by comparing with the finite-difference method and the analytical solution. Moreover, using the MRT-LB method coupled with the linear driving force model, the fluid transfer and adsorption behaviors of the carbon dioxide in a porous fixed bed are explored. The breakthrough curve of adsorption from MRT-LB simulation is compared with the experimental data and the finite-element solution, and the transient concentration distributions of the carbon dioxide along the porous fixed bed are elaborated in detail. In addition, the MRT-LB simulation results show that the appearance time of breakthrough point in breakthrough curve is advanced as the mass transfer resistance in linear driving force model increases, however, the saturation point is prolonged inversely.

\footnotetext{
*zqchen@seu.edu.cn

\#liu.hao@nottingham.ac.uk
} 


\section{INTRODUCTION}

The problem of flow and mass transfer with adsorption/desorption phenomena in porous media is ubiquitous and significant in many fields of science and engineering, including carbon capture and storage technology [1], environmental pollution improvement [2] and chemical engineering [3]. To fully understand this process, an appropriate mathematical model is necessary for the theoretical description and will help to optimize the flow and mass transfer with adsorption processes in the equipment (such as a porous adsorbent bed) [4]. In general, to predict the complete dynamical behaviours in porous media, a coupling mathematical model, including momentum-, mass-, and energy-conservation equations, need to be constructed simultaneously[5]. In addition, for describing the effect of the adsorption behaviours on the mass transfer process, an appropriate mass transfer kinetic model of adsorption should be incorporated into the mass-conservation equation as a source term [6-8]. It is worth to note that the solving processes of this coupling model are tedious and time-consuming [9]. In this context, the development of a simplified and efficient numerical model for predicting the flow and adsorption processes in porous media has attracted much attention in recent years.

As a promising mesoscopic numerical method, the lattice Boltzmann (LB) model has some significant advantages over the conventional numerical methods in modeling complex physics in fluids, such as its role as a linear convective operator, its high efficiency for parallel performance, and its excellent applicability to cases with 
complicated boundary conditions [10-12]. Consequently, the LB model has rapidly emerged as a powerful numerical tool in studies involving simulations of momentum-, heat- and mass-transport problems [13-20], including transport phenomena in porous media. Generally, the LB simulation in porous media involves micro-scale (pore scale), and meso-scale (representative elementary volume scale, REV scale) [13], where the relationship between the two scales is illustrated in Fig.1. At the microscopic pore scale, the geometric structure of porous media is described in detail; while, at the mesoscopic REV scale, the geometric structure is simplified by an elementary cell using the volume-averaging method. Due to the briefness of boundary schemes to handle the complex morphology of porous matrix, a series of pore scale LB models have been proposed to study the adsorbate's mass transport and adsorption behaviors in the pore structures of porous media, which were constructed by the regular particles or the stochastic irregular solid matrix[21-28]. By using these LB simulations at the pore scale, the transient fluid velocity and the adsorbate concentration profiles in the pore structures were presented in detail, and the effect mechanism of pore structure characteristics on the dynamic adsorption performance was elaborated to provide a guidance for the design and optimization of adsorption systems in the micro-scale. Nevertheless, the present pore scale studies need to describe the detailed geometric information of porous structures, thus the size of computation domain cannot be too large due to the limited computer resources[29].

In contrast, because the geometric structure of porous media is ignored, the REV scale numerical study can overcome the limitation of computation domain at the pore 
scale, and hence can be used for systems with the large computation domain [30]. In recent years, the REV LB model, as a computationally efficient numerical method [31], has been successfully used in the simulation of flow and heat transfer in porous media[32-34]. Moreover, to address some inherent shortcomings of the singlerelaxation-time LB model, such as the numerical inaccuracy and the instability problems, the multiple-relaxation-time (MRT) LB model, as an effective improving scheme[35], has been employed to solve the transfer problems in porous media at the REV scale more accurately[13,36]. However, to the best of our knowledge, little work has been reported on the application of the LB model coupled with a mass transfer kinetic model of adsorption in solving mass transfer with adsorption problems in porous media at the REV scale.

Hence, the present work aims at developing an MRT-LB model to explore the flow, mass transfer and adsorption behaviours in porous media at the REV scale. This model includes an MRT-LB model for the fluid flow and an MRT-LB model for the mass transfer with adsorption. In Section II, the macroscopic governing equations of fluid flow, mass transfer and adsorption processes in porous media are first presented. In Section IIIA, an existing MRT-LB model for momentum transfer in porous media is introduced to solve the fluid flow in porous media at the REV scale. In Section IIIB, based on the work of LB model for the convection-diffusion equation (CDE) in nonporous media[37,38], an MRT-LB model is developed to simulate the mass transfer with an unsteady source term in porous media at the REV scale. In Section IV, the combined MRT-LB model is validated by comparing it with the finite-difference 
method and the analytical solution. Then, the linear driving force (LDF) model, as a widely used mass transfer kinetics model of adsorption process[39-43], is considered as an unsteady source term in the LB model for the CDE to describe the adsorption behaviour of the adsorbate. The above modified MRT-LB model is used to simulate the carbon dioxide flow, mass transfer and adsorption processes in a porous fixed bed of activated carbon, and the numerical results are compared with the experimental data and the finite-element solution. Finally, some conclusions are given in Section V.

\section{Macroscopic governing equations}

For the fluid flow and mass transfer coupled with the adsorption process in homogeneous, isotropic porous media, the macroscopic momentum transfer at the REV scale, which is governed by the generalized Navier-Stokes equations, can be expressed as[31]:

$$
\begin{gathered}
\nabla \cdot \mathbf{u}=0 \\
\frac{\partial \mathbf{u}}{\partial t}+(\mathbf{u} \cdot \nabla)\left(\frac{\mathbf{u}}{\varepsilon}\right)=-\frac{1}{\rho_{0}} \nabla(\varepsilon p)+v_{e} \nabla^{2} \mathbf{u}+\mathbf{F},
\end{gathered}
$$

where $\boldsymbol{u}$ and $p$ denote the volume-averaged fluid velocity and pressure, respectively; $\rho_{0}$ is the mean fluid density; $\varepsilon$ denotes the porosity of the porous media; $v_{e}$ is the effective kinetic viscosity; and $\boldsymbol{F}$ is the total body force.

Considering an adsorbate in the fluid, the governing equation of the mass transfer of adsorbate can be written as the CDE with a source term in porous media[44]:

$$
\varepsilon \frac{\partial C}{\partial t}+\nabla(\mathbf{u} C)=\varepsilon D \nabla^{2} C+R^{*}
$$

where $C$ is the adsorbate concentration in the fluid, $D$ is the effective diffusion coefficient of the adsorbate in porous media, and $R^{*}$ is an unsteady source term that 
represents the rate of mass transfer through adsorption by the absorbent particle of the porous media. Based on the LDF model, $R^{*}$ is given by [9]

$$
R^{*}=(1-\varepsilon) \rho_{b} \frac{\partial \bar{q}}{\partial t}=(1-\varepsilon) \rho_{b} K_{L}\left(q^{*}-\bar{q}\right)
$$

where $\bar{q}$ denotes the average amount adsorbed by the adsorbent particle, $q^{*}$ represents the amount adsorbed at equilibrium with the instantaneous adsorbate partial pressure, $\rho_{b}$ is the adsorbent particle density, and $K_{L}$ is the effective LDF mass transfer coefficient.

In addition, the total body force of fluid $\boldsymbol{F}$ can be written as:

$$
\mathbf{F}=-\frac{\varepsilon v}{K} \mathbf{u}-\frac{\varepsilon F_{\phi}}{\sqrt{K}}|\mathbf{u}| \mathbf{u}+\varepsilon \mathbf{a}
$$

where $v$ denotes the kinetic viscosity, $K$ represents the permeability, $F_{\phi}$ is the geometric function, and $\boldsymbol{a}$ is the external force. Using Ergun's relation, the geometric function and the permeability of the porous media are given by

$$
F_{\phi}=\frac{1.75}{\sqrt{150 \varepsilon^{3}}}, \quad K=\frac{\varepsilon^{3} \mathrm{~d}_{p}^{2}}{150(1-\varepsilon)^{2}}
$$

where $d_{p}$ is the mean diameter of the adsorbent particles. The key parameters of the fluid flow and adsorption process can be characterized using several dimensionless numbers, including the Darcy number $D a$, the Reynolds number $R e$, the Schmidt number $S c$, the Fourier number $F o$, and the dimensionless ratio $K^{*}$ of the LDF mass transfer coefficient to the diffusion coefficient, which are defined as:

$$
\begin{aligned}
& D a=\frac{K}{L^{2}}, \quad R e=\frac{L U}{v}, \quad S c=\frac{v}{D} . \\
& F o=\frac{D \tau}{L^{2}}, \quad K^{*}=\frac{K_{L} L^{2}}{D}
\end{aligned}
$$

where $L$ and $U$ are the characteristic length and velocity of the system, respectively, and $\tau$ is the computation time for the fluid flow and mass transfer process. 


\section{MRT-LBM for the flow, mass transfer and adsorption processes in porous media}

\section{A. MRT-LBM for momentum transfer in porous media}

To simulate the fluid flow in porous media using the LB method, an existing D2Q9 MRT-LB model [36] is adopted to solve the generalized Navier-Stokes equations, given as Eq. (1) and Eq. (2). The evolution equation with a forcing term can be expressed as:

$$
f_{\mathrm{i}}\left(\boldsymbol{x}+\boldsymbol{e}_{i} \delta t, t+\delta t\right)-f_{\mathrm{i}}(\boldsymbol{x}, t)=\left(\boldsymbol{M}^{-1} \boldsymbol{\Gamma} \boldsymbol{M}\right)_{i j}\left[f_{\mathrm{j}}(\boldsymbol{x}, t)-f_{\mathrm{j}}^{e q}(\boldsymbol{x}, t)\right]+\boldsymbol{M}^{-1} \delta t\left(\boldsymbol{I}-\frac{\boldsymbol{\Gamma}}{2}\right) \boldsymbol{S},
$$

where $f_{i}(\boldsymbol{x}, \boldsymbol{t})$ denotes the volume-averaged density distribution function associated with the discrete velocity $\boldsymbol{e}_{\mathrm{i}}$ at position $\boldsymbol{x}$ and time $t, \delta t$ is time step, $\boldsymbol{I}$ is the unit matrix, $\boldsymbol{M}$ is a 9x9 orthogonal transformation matrix, $\Gamma$ is a diagonal relaxation matrix, which is related to the collision matrix, and the forcing term $S$ is a 9-dimensional column vector:

$$
\begin{gathered}
\mathbf{M}=\left(\begin{array}{ccccccccc}
1 & 1 & 1 & 1 & 1 & 1 & 1 & 1 & 1 \\
-4 & -1 & -1 & -1 & -1 & 2 & 2 & 2 & 2 \\
4 & -2 & -2 & -2 & -2 & 1 & 1 & 1 & 1 \\
0 & 1 & 0 & -1 & 0 & 1 & -1 & -1 & 1 \\
0 & -2 & 0 & 2 & 0 & 1 & -1 & -1 & 1 \\
0 & 0 & 1 & 0 & -1 & 1 & 1 & -1 & -1 \\
0 & 0 & -2 & 0 & 2 & 1 & 1 & -1 & -1 \\
0 & 1 & -1 & 1 & -1 & 0 & 0 & 0 & 0 \\
0 & 0 & 0 & 0 & 0 & 1 & -1 & 1 & -1
\end{array}\right) \\
\Gamma=\operatorname{diag}\left(\gamma_{0}, \gamma_{1}, \cdots, \gamma_{8}\right), \\
\quad \boldsymbol{S}=\left(S_{0}, S_{1} \cdots, S_{8}\right)^{T},
\end{gathered}
$$

where $\gamma_{i}\left(0<\gamma_{i}<2\right)$ is the relaxation rate and $S_{i}$ is a component of $S$, which will be determined later. For the D2Q9 model, the discrete velocity $\boldsymbol{e}_{i}$ is given by

$$
\boldsymbol{e}_{i}=\left\{\begin{array}{cc}
(0,0) & i=0 \\
c\left(\cos \left[(i-1) \frac{\pi}{2}\right], \sin \left[(i-1) \frac{\pi}{2}\right]\right) & i=1,2,3,4, \\
\sqrt{2} c\left(\cos \left[(2 i-9) \frac{\pi}{4}\right], \sin \left[(2 i-9) \frac{\pi}{4}\right]\right) & i=5,6,7,8
\end{array}\right.
$$

where $c=\delta x / \delta t$ is the lattice speed with $\delta x$ denoting the lattice spacing step and $\delta x$ is set 
equal to $\delta t$ in this paper. The equilibrium distribution function is expressed as:

$$
f_{i}^{\mathrm{eq}}=\left\{\begin{array}{l}
\rho_{0}-\left(1-\omega_{0}\right) \frac{\varepsilon p}{c_{s}^{2}}+\rho_{0} s_{0}(\boldsymbol{u}), \quad i=0 \\
\omega_{i} \frac{\varepsilon p}{c_{s}^{2}}+\rho_{0} s_{i}(\boldsymbol{u}), \quad i=1,2, \ldots 8
\end{array},\right.
$$

where $\omega_{i}$ is the weight coefficient of the D2Q9 model with $\omega_{0}=4 / 9, \omega_{i}=1 / 9$ for $i=1$ $-4, \omega_{i}=1 / 36$ for $i=5-8, c_{s}=1 / \sqrt{3}$ represents the lattice sound speed, and $s_{i}(\boldsymbol{u})$ is given by

$$
s_{i}(\boldsymbol{u})=\omega_{\mathrm{i}}\left[\frac{\boldsymbol{e}_{i} \cdot \boldsymbol{u}}{c_{s}^{2}}+\frac{\left(\boldsymbol{e}_{i} \cdot \boldsymbol{u}\right)^{2}}{2 \varepsilon c_{s}^{4}}-\frac{|\boldsymbol{u}|^{2}}{2 \varepsilon c_{s}^{2}}\right]
$$

In this LB model, the total body force of fluid $\boldsymbol{F}$ in Eq. (2) is represented using the forcing term $\boldsymbol{S}$. Based on the work of Liu et al.[36], the components of $\boldsymbol{S}$ are chosen as:

$$
\begin{array}{ll}
S_{0}=0, \quad S_{1}=\frac{6 \rho_{0} \boldsymbol{u} \cdot \boldsymbol{F}}{\varepsilon}, & S_{2}=-\frac{6 \rho_{0} \boldsymbol{u} \cdot \boldsymbol{F}}{\varepsilon}, \\
S_{3}=\rho_{0} F_{x}, \quad S_{4}=-\rho_{0} F_{x}, & S_{5}=\rho_{0} F_{y}, \quad S_{6}=-\rho_{0} F_{y}, \\
S_{7}=\frac{2 \rho_{0}\left(u_{x} F_{x}-u_{y} F_{y}\right)}{\varepsilon}, & S_{8}=\frac{2 \rho_{0}\left(u_{x} F_{y}+u_{y} F_{x}\right)}{\varepsilon} .
\end{array}
$$

The macroscopic variables, including the volume-averaged fluid velocity $\boldsymbol{u}$ and pressure $p$, are defined as[31]:

$$
\begin{gathered}
\mathbf{u}=\frac{\mathbf{v}}{l_{0}+\sqrt{l_{0}^{2}+l_{1}|\mathbf{v}|}}, \\
p=\frac{c_{s}^{2}}{\varepsilon\left(1-\omega_{0}\right)}\left[\sum_{i=0}^{8} \boldsymbol{e}_{i} f_{i} / \rho_{0}+\rho_{0} \mathrm{~s}_{0}(\boldsymbol{u})\right],
\end{gathered}
$$

where $v$ is a temporal velocity and $l_{0}$ and $l_{l}$ are two parameters of the model, which are given by

$$
\begin{aligned}
& \mathbf{v}=\sum_{i=0}^{8} \mathbf{e}_{i} f_{i} / \rho_{0}+\frac{\delta t}{2} \varepsilon \mathbf{a}, \\
& l_{0}=\frac{1}{2}\left(1+\varepsilon \frac{\delta t}{2} \frac{v}{K}\right),
\end{aligned}
$$




$$
l_{1}=\varepsilon \frac{\delta t}{2} \frac{F_{\phi}}{\sqrt{K}}
$$

In addition, the effective kinetic viscosity $v_{e}$ is defined as:

$$
v_{\mathrm{e}}=c_{s}^{2}\left(\frac{1}{\gamma_{7}}-\frac{1}{2}\right) \delta_{t}=c_{s}^{2}\left(\frac{1}{\gamma_{8}}-\frac{1}{2}\right) \delta_{t} .
$$

Based on the Chapman-Enskog analysis [45], the continuity equation Eq. (1) and the momentum equation Eq. (2) can be recovered from this MRT-LB model.

\section{B. MRT-LBM for the convection-diffusion equation in porous media}

In addition to the above MRT-LB model for the momentum transfer in porous media, one other appropriate MRT-LB model should be constructed to simulate the mass transfer coupled with the adsorption process, as described in Eq. (3), which is a typical CDE in porous media. In recent years, a series of LB models for the $\mathrm{CDE}[37,38,46]$ were developed to investigate the mass transfer or heat transfer problems of fluids. While, there is relatively less work on MRT-LB models for solving mass transfer with a source term in porous media. To eliminate this research gap, inspired by the idea of Chai et al.[37], we develop an MRT-LB model for the CDE with a source term in porous media at the REV scale, and Eq. (3) can be recovered from the modified model using Champan-Enskog analysis. The evolution equation of the MRTLB model for the CDE can be expressed as:

$$
\mathrm{g}_{\mathrm{i}}\left(\boldsymbol{x}+\boldsymbol{e}_{i} \delta t, t+\delta t\right)-\mathrm{g}_{\mathrm{i}}(\boldsymbol{x}, t)=\Omega_{i}+\delta t G_{i}
$$

where $g_{i}(\mathrm{x}, \mathrm{t})$ is the discrete distribution function of a scalar variable. For the mass transfer problem governed by the $\mathrm{CDE}$, the scalar variable represents the concentration variable $C$, and $g_{i}(\mathrm{x}, \mathrm{t})$ denotes the concentration distribution function, which is 
different from $f_{i}(\boldsymbol{x}, \boldsymbol{t})$ representing the density distribution function. On the right-hand side of Eq. (22), the collision term is given by

$$
\Omega_{i}=-\left(\mathbf{M}^{-1} \Lambda \mathbf{M}\right)_{i j}\left[\mathrm{~g}_{\mathrm{j}}(\mathbf{x}, t)-\mathrm{g}_{\mathrm{j}}^{e q}(\mathbf{x}, t)\right],
$$

where the equilibrium distribution is modified as:

$$
\mathrm{g}_{\mathrm{j}}^{e q}(\boldsymbol{x}, t)=\omega_{\mathrm{i}} C\left[\varepsilon+\frac{\boldsymbol{e}_{\boldsymbol{i}} \cdot \boldsymbol{u}}{c_{s}^{2}}+\frac{\left(\boldsymbol{e}_{\boldsymbol{i}} \cdot \boldsymbol{u}\right)^{2}}{2 \varepsilon c_{s}^{4}}-\frac{\boldsymbol{e}_{\boldsymbol{i}} \cdot \boldsymbol{u}}{2 \varepsilon c_{s}^{2}}\right]+\eta_{i} \frac{C \varepsilon p}{c_{s}^{2} \rho_{0}},
$$

and $\eta_{i}$ denotes the coefficients that are given by

$$
\eta_{\mathrm{i}}=\omega_{i}(i \neq 0), \quad \eta_{0}=-\sum_{\mathrm{i} \neq 0} \eta_{\mathrm{i}}
$$

where $\eta_{i}$ is the weight coefficient of the D2Q9 model, with $\eta_{0}=-5 / 9, \eta_{i}=1 / 9$ for $i=1$ to 4 , and $\eta_{i}=1 / 36$ for $i=5$ to 8 . The diagonal relaxation matrix is given by

$$
\Lambda=\operatorname{diag}\left(\lambda_{0}, \lambda_{1}, \cdots, \lambda_{8}\right) \text {, }
$$

where $0<\lambda_{i}<2$. Considering the effect of the porosity of the porous media, the source term of the MRT-LB model is constructed as:

$$
\begin{gathered}
G_{i}=\left[\mathbf{M}^{-1}\left(\mathbf{I}-\frac{\boldsymbol{\Lambda}}{2}\right) \mathbf{M}\right]_{i j} R_{j}, \\
R_{\mathrm{i}}=\omega_{\mathrm{i}}\left[\left(1+\frac{\boldsymbol{e}_{i} \cdot \boldsymbol{u}}{\varepsilon c_{s}^{2}}\right) R+\frac{\boldsymbol{e}_{i} \cdot\left(\varepsilon p \nabla C / \rho_{0}+C \boldsymbol{F}\right)}{c_{s}^{2}}\right]
\end{gathered}
$$

where $R$ is the source term of the macroscopic CDE. Using the transformation matrix $\boldsymbol{M}$, the discrete concentration distribution function and the equilibrium distribution function can be projected onto their macroscopic variables in the moment space:

$$
\boldsymbol{m}:=\boldsymbol{M g}, \quad \boldsymbol{m}^{(e q)}:=\boldsymbol{M g}^{(e q)},
$$

where $\boldsymbol{m}=\left(m_{0}, m_{1}, \ldots m_{8}\right)^{\mathrm{T}}$ and $\left.\boldsymbol{m}^{(e q)}=\left(m^{(e q)}{ }^{0}, m^{(e q) 1}, \ldots m^{(e q)}\right)^{\mathrm{T}}\right)^{\mathrm{T}}$. The scalar variable $C$ can be obtained using Eq. (30): 


$$
\varepsilon C=\sum_{i} \mathrm{~g}_{i}+\frac{\delta t}{2} R
$$

Using the Champan-Enskog analysis (the details are presented in Appendix A), Eq. (3) will be recovered from the developed LB model. Hence, this model can be used to solve the mass transfer with absorption in porous media at the REV scale, which is different from the previous MRT-LB model for CDE in non-porous media [37]. In addition, based on the nonequilibrium scheme [38], the flux can be calculated as follows:

$$
\boldsymbol{J}=D \frac{\sum_{i} \boldsymbol{c}_{i}\left[g_{i}-g_{i}^{e q}\right]+\frac{\delta t}{2}\left(C \boldsymbol{F}+\boldsymbol{u} \frac{R}{\varepsilon}\right)}{\varepsilon \delta t\left(\frac{c_{s}^{2}}{\lambda_{3}}+\frac{p}{2 \rho_{0}}\right)}+C \boldsymbol{u} .
$$

It is noteworthy that the MRT-LB model is not only used to investigate the mass transfer coupled with adsorption in porous media, but also applied to study heat or mass transfer as the convection-diffusion problem in porous media when the scalar variable and the source term are adjusted appropriately.

\section{Treatment of boundary and initial conditions}

The boundary conditions are critical for the accurate implementation of the LB model. In this paper, as a method of second-order accuracy, the non-equilibrium extrapolation scheme [47] is employed to treat the boundary conditions for the MRTLB model. For the LB model of the momentum transfer, the distribution function at the boundary node $\boldsymbol{x}_{b}$ can be expressed as :

$$
f_{\mathrm{i}}\left(\boldsymbol{x}_{b}, t\right)=f_{\mathrm{i}}^{e q}\left(\boldsymbol{x}_{b}, t\right)+\left[f_{\mathrm{i}}\left(\boldsymbol{x}_{f}, t\right)-f_{\mathrm{i}}^{e q}\left(\boldsymbol{x}_{f}, t\right)\right],
$$

where $\boldsymbol{x}_{f}$ denotes the nearest-neighbour fluid node of $\boldsymbol{x}_{b}\left(\boldsymbol{x}_{f}=\boldsymbol{x}_{b}+\boldsymbol{e}_{\mathrm{i}} \delta t\right)$. For the velocity boundary condition, such as the no-slip wall boundary, the unknown pressure value can 
be substituted by the pressure at the nearest-neighbour fluid node, and the equilibrium distribution function of the boundary node in Eq. (32) can be calculated as follows:

$$
f_{i}^{\mathrm{eq}}\left(x_{b}, t\right)=\left\{\begin{array}{l}
\rho_{0}-\left(1-\omega_{0}\right) \frac{\varepsilon p\left(\boldsymbol{x}_{f}, t\right)}{c_{s}^{2}}+\rho_{0} s_{0}\left(\boldsymbol{u}\left(x_{b}, t\right)\right), \quad i=0 \\
\omega_{i} \frac{\varepsilon p\left(\boldsymbol{x}_{f}, t\right)}{c_{s}^{2}}+\rho_{0} s_{i}\left(\boldsymbol{u}\left(x_{b}, t\right)\right), \quad i=1,2, \ldots 8
\end{array} .\right.
$$

As such, the distribution function at the boundary node for the CDE can be calculated as:

$$
g_{\mathrm{i}}\left(\boldsymbol{x}_{b}, t\right)=g_{\mathrm{i}}^{e q}\left(\boldsymbol{x}_{b}, t\right)+\left[g_{\mathrm{i}}\left(\boldsymbol{x}_{f}, t\right)-g_{\mathrm{i}}^{e q}\left(\boldsymbol{x}_{f}, t\right)\right]
$$

For the Dirichlet boundary condition, the equilibrium distribution function at the boundary node can be obtained using Eq. (24). However, for the Neumann boundary condition, the unknown scalar variable $C\left(x_{b}, \mathrm{t}\right)$ should be estimated based on the gradient of the scalar variable at the boundary node[36]:

$$
C\left(\boldsymbol{x}_{b}, t\right)=\frac{4 C\left(\boldsymbol{x}_{f}, t\right)-C\left(\boldsymbol{x}_{f f}, t\right)-2 \Delta \cdot \nabla C\left(\boldsymbol{x}_{b}, t\right)}{3},
$$

where the $\boldsymbol{x}_{f f}$ denotes the nearest-neighbour fluid node of $\boldsymbol{x}_{f}$, and $\Delta=\boldsymbol{x}_{f}-\boldsymbol{x}_{b}=\boldsymbol{x}_{f f}-\boldsymbol{x}_{f}$, and the gradient of $C\left(\boldsymbol{x}_{b}, \mathrm{t}\right)$ is obtained using the Fick law $\left(\boldsymbol{J}_{b}=-D \nabla C\left(\boldsymbol{x}_{b}, \mathrm{t}\right)\right)$, where the flux at the boundary $\boldsymbol{J}_{\boldsymbol{b}}$ is known for the Neumann boundary condition. In addition, at the initial time of the simulation, the distribution function is obtained from its equilibrium distribution function:

$$
f_{\mathrm{i}}(\boldsymbol{x}, t=0)=f_{\mathrm{i}}^{e q}(\boldsymbol{x}, t=0), \quad g_{\mathrm{i}}(\boldsymbol{x}, t=0)=g_{\mathrm{i}}^{e q}(\boldsymbol{x}, t=0) .
$$

\section{RESULTS AND DISCUSSION}

\section{A. Numerical tests for the MRT-LB model}


In this section, the proposed method is evaluated on several benchmarks, and the results are compared with those of the finite-difference method and the analytical solution to validate the proposed MRT-LB model for simulating the flow and convection-diffusion problems with a source term in porous media. In benchmark 1, the isothermal Poiseuille flow in a $2 \mathrm{D}$ porous channel is investigated to demonstrate the effectiveness of the MRT-LBM for momentum transfer. Then, considering the comparability for heat transfer and mass transfer problems described by the CDE, the applicability of the MRT-LBM for the CDE in porous media is clarified by the simulation of thermal Poiseuille flow with heat dissipation in benchmark 2. In addition, to examine the validity of the MRT-LB simulation combined with the unsteady LDF model, the unsteady mass diffusion process is studied using the MRT-LBM for the CDE with an unsteady source term in benchmark 3 .

\section{Poiseuille flow in a $2 D$ porous channel}

Guo and Zhao[31] simulated the Poiseuille flow in a 2D channel filled with porous media to confirm the validity of the LBGK model for the flow field in porous media. Similarly, the numerical results calculated by this adopted MRT-LB momentum model are compared with the solutions obtained using the finite-difference method (FDM) from Guo and Zhao's work.

The schematic of the Poiseuille flow in a 2D porous channel is shown in Fig. 2. The height of the channel $H$ is selected as the characteristic length, and the fluid is driven using an external force along the x-axis direction $\boldsymbol{a}_{x}$. The inlet and outlet of 2D channel are set as the periodic boundary, and the no-slip velocity boundary is employed 
at the top and bottom walls using the non-equilibrium extrapolation scheme.

In this validation case, the porosity of the porous channel $\varepsilon$ is 0.1 , the viscosity ratio $v_{e} / v$ is set as 1 , and the grid size $(L \times H)$ is $80 \times 80$. The relaxation rates are chosen as $\gamma_{0=} \gamma_{3}=\gamma_{5}=1, \gamma_{1}=\gamma_{2}=1.1$, and $\gamma_{4}=\gamma_{6}=1.2$, while $\gamma_{7}$ and $\gamma_{8}$ are set to be $5 / 3$. At the initial time $(F o=0)$, the velocity field in the computational domain is initialized as 0 . Using the MRT-LB for momentum model, the velocity distributions of the fully developed Poiseuille flow along the y-axis direction are investigated under different Darcy numbers and Reynolds numbers, where the characteristic velocity $U_{0}$ in Reynolds number is the maximum velocity at the centreline of the $2 \mathrm{D}$ porous channel without considering the nonlinear Forchheimer drag force. Fig. 3(a) shows the dimensionless velocity distributions at a given Darcy number $D a=10^{-5}$. It is found that the fluid peak velocity is reduced when the Reynolds number $R e$ varies from 0.01 to 100 . This is caused by the increased nonlinear drag force with increased $R e$. For the different Reynolds numbers, the numerical results of the MRT-LB model agree with the solutions of the FDM very well. On the other hand, when the Darcy number $D a$ varies from $10^{-6}$ to $10^{-3}$ at a given Reynolds number $(\operatorname{Re}=0.1)$, the effect of the resistance of the porous media on the Poiseuille flow is presented in Fig. 3(b), and the velocity profiles predicted by the adopted LB model are in excellent agreement with the solutions of the FDM.

\section{Thermal Poiseuille flow with heat dissipation}

To validate the developed MRT-LBM for the CDE with a source term, the thermal Poiseuille flow in a $2 \mathrm{D}$ channel with heat dissipation is simulated based on Chai and Zhao's work[37]. Note that the scalar variable $C$ in the CDE denotes the 
temperature $T$ provisionally, and the source term $R$ represents the viscous heat dissipation, which is defined as

$$
R=\frac{2 v}{C_{v}}(\mathbf{S}: \mathbf{S})
$$

$$
\text { where } \mathbf{S}=\left[\nabla \mathbf{u}+(\nabla \mathbf{u})^{T}\right] / 2,
$$

and $C_{v}$ denotes the specific heat at constant volume. As the thermal Poiseuille flow includes the temperature field, the temperature at the top wall and at the bottom wall are set as $T_{0}=1.1$ and $T_{1}=0.1$, respectively. The periodic temperature boundary is employed at the inlet and outlet of the 2D channel. In addition, the momentum transfer is achieved using the above MRT-LBM for the momentum transfer, and the boundary conditions have been described in the above subsection, and the grid size $(L \times H)$ is also $80 \times 80$. As a steady problem, we can consider the numerical results to have reached the steady state when the following condition is satisfied:

$$
\frac{\sum_{x}|T(\boldsymbol{x}, t)-T(\boldsymbol{x}, t-\delta t)|}{\sum_{x} T(\boldsymbol{x}, t)}<1.0 \times 10^{-8} .
$$

In the first stage, the effect of porosity is neglected $(\varepsilon=1)$, and the fluid is driven by an external force along the x-axis direction $\boldsymbol{a}_{x}$. The temperature and heat flux profiles are calculated using the proposed MRT-LB model as the Prandtl number $\operatorname{Pr}=v / D=1$ and the defined Froude number $F r=U_{\max }^{2} / \boldsymbol{a}_{x} H=14.7$, where $U_{\max }$ denotes the maximum velocity at the centerline of the $2 \mathrm{D}$ channel.

In the numerical results as shown in Fig. 4, the dimensionless temperature $\Theta_{T}$ is defined as $\Theta_{T}=\left(T-T_{1}\right) /\left(T_{0}-T_{1}\right)$, and the dimensionless heat flux $J^{*}$ is defined as: 


$$
\boldsymbol{J}^{*}=\frac{\boldsymbol{J} \cdot H}{D\left(T_{0}-T_{1}\right)} .
$$

It is found that the dimensionless temperature and the dimensionless heat flux are enhanced by the increasing heat dissipation indicated by the Eckert number $(E c)$, and $E c=U_{m a}^{2}\left\{C_{v}\left(T_{0}-T_{1}\right)\right]$. When the Eckert number varies from 10 to 100 , we can see that the numerical results are agree well with the analytical solutions, which are clarified in Appendix $\mathrm{B}$, and the maximum error in the temperature between the numerical and analytical solutions is less than $1 \%$ at $E c=100$.

In what follows, the proposed MRT-LBM for the CDE is applied to simulate the thermal Poiseuille flow in a 2D porous channel. For the Poiseuille flow conditions identical to those presented in Fig. 3(b) $(\varepsilon=0.1, R e=0.1)$, the constant temperatures $T_{0}=1.1$ and $T_{l}=0.1$ are employed at the top wall and bottom wall, respectively. If the source term is not considered, the profiles of temperature and heat flux, as the Darcy number $D a$ varies from $10^{-6}$ to $10^{-3}$, can be calculated using the FDM. The comparative results between the MRT-LBM and the FDM are shown in Fig. 5. Due to the absence of effects of heat dissipation and convection, the profiles of dimensionless temperature vary linearly from 0 to 1 , governed by Fick's law. But, the profiles of dimensionless heat flux along the $x$-axis are significantly different for different $D a$ numbers, which is caused by the varying distribution of velocity with the different permeability. In addition, the numerical results from the proposed MRT-LB model are in excellent agreement with the solutions of the FDM.

\section{Mass diffusion with an unsteady state source term}

In this subsection, the MRT-LB model is used to calculate the CDE coupled with 
an unsteady state source term, which is similar to the source term of Eq. (3), governed by the LDF model. To validate the MRT-LB model with an unsteady source term through comparison with an analytical method, the fluid flow is ignored in this case $(R e=0)$, and a constant concentration $C_{0}=0$ is employed at the top wall and bottom wall of the 2D porous channel $(\varepsilon=0.1)$, as shown in Fig. 2. It is important to note that the scalar variable $C$ of the $\mathrm{CDE}$ denotes the concentration hereafter. The mass source term $R$ is defined as:

$$
R=k_{m}\left(C_{1}-C\right)
$$

where $k_{m}$ is a mass transfer coefficient, $C_{l}$ is a constant concentration $C_{l}=1$, and $C$ is the transient concentration in the computational domain, which is set as $C_{0}$ at the initial time $\tau=0$. The grid size for this numerical simulation $(L \times H)$ is $60 \times 60$, and the dimensionless concentration $\Theta_{C}$ is defined as $\Theta_{C}=\left(C-C_{0}\right) /\left(C_{1}-C_{0}\right)$. As simulation results, the profiles of the dimensionless transient concentration with different dimensionless mass transfer coefficients are plotted in Fig. 6. It is obvious that the concentration increases with increasing dimensionless time due to the mass source term. When the dimensionless mass transfer coefficient $k_{m}^{*}\left(k_{m}^{*}=k_{m} H^{2} / D\right)$ rises from 0.72 to 36 , the time to reach the steady state is reduced, and the equilibrium concentration is closer to the constant concentration $C_{1}$. Moreover, for the different dimensionless mass transfer coefficients and the dimensionless time, the numerical results from the MRT-LB model are consistent with the analytical solutions, which are explained in Appendix C.

\section{B. MRT-LB simulation of carbon dioxide flow, mass transfer and adsorption in a porous fixed bed}


Based on the above validation work for the MRT-LB model, the proposed model is applied to simulate the transfer and adsorption processes of carbon dioxide from nitrogen as a carrier gas in a porous fixed bed of activated carbon. Due to the independent of adsorption behaviours of carbon dioxide and nitrogen [48], carbon dioxide is considered as a single adsorbate in this work. To simplify the problem, the porous fixed bed is viewed as a $2 \mathrm{D}$ channel with length $L$, as shown in Fig. 7 . The boundary conditions of the N-S equations for the flow of gas mixtures are identical to those described for the Poiseuille flow in Section IV A. Meanwhile, the concentration of carbon dioxide at the inlet of the channel is set as $C_{0}$ and is given by Eq. (42):

$$
\mathbf{u} C_{f}=\mathbf{u} C_{0}-\varepsilon D \nabla_{x} C,
$$

where $C_{f}$ denotes the feed concentration, which is a constant. Moreover, the concentration gradient at the outlet is set to zero, and non-permeable boundaries (the mass flux is zero) are employed at the top and bottom walls. Unless otherwise stated, the non-equilibrium extrapolation scheme is adopted to treat these boundary conditions of the CDE for the mass transfer of carbon dioxide. At the initial time, the concentration of carbon dioxide is equal to zero in the fixed bed.

In addition, to utilize the LDF model for the adsorption behaviour of carbon dioxide as a source term in the CDE; the amount adsorbed at equilibrium, described by Eq. (A22), should be determined using the isotherm Toth equation for singlecomponent adsorption[48,49]:

$$
q^{*}=\frac{q_{m} K_{e q} p_{c}}{\left[1+\left(K_{e q} p_{c}\right)^{\varphi}\right]^{1 / \varphi}}
$$

where $q_{m}$ is the maximum adsorbed concentration, $\varphi$ is the heterogeneity parameter, $p_{c}$ 
is the partial pressure of carbon dioxide, which is obtained from the concentration of carbon dioxide as an ideal gas, and the equilibrium adsorption constant $K_{e q}$ is determined by Eq. (44):

$$
K_{e q}=K_{e q}^{0} \exp \left(\frac{-\Delta \mathrm{H}}{R_{0} T}\right)
$$

where $-\Delta H$ is the adsorption enthalpy and $R_{0}$ is the gas constant. According to the work of Dantas et al. [44,48], the parameters in Eqs. (43) and (44) are given in Table I.

Since the fluid transfer process occurs in a long 2D porous channel, the effect of the concentration distribution along the width direction can be neglected. Therefore, the flow and adsorption studied in this work can be regarded approximately as a onedimensional transport process along the length direction, and the length $L$ of the channel acts as the characteristic length. For comparison with the experimental and finiteelement method results at temperature $T=423 \mathrm{~K}$ from the literature[44], the physical and transport properties of the fluid and porous fixed bed are determined using a series of dimensionless numbers, which are listed in Table II.

The transient concentration at the outlet of porous fixed bed is plotted in Fig. 8 as a typical breakthrough curve. It is found that the concentration of outlet remains constant at zero until the time of the breakthrough point is reached, which indicates that the influent carbon dioxide is completely adsorbed by the porous fixed bed at the early stages of the adsorption process. Then the transient concentration of outlet rises to the feed concentration sharply until the time of the saturation point is reached, which indicates the adsorption process has been completed. For the most part, the LB model result for the outlet well agreed with the experimental data and the finite-element 
solution from the literature[44]. The comparison result shows that this developed LB model, as an efficient mesoscopic numerical method, can be used to simulate the flow, mass transfer and adsorption process in porous adsorbent equipment at the REV scale.

Furthermore, the mole flux distribution and transient concentration along the length direction are shown in Fig. 9, where the dimensionless mole flux is defined as

$$
J^{*}=J_{x} L /\left(C_{f} \cdot D\right)
$$

At the early stages of the adsorption process $(F O=0.08)$, because most of the carbon dioxide is adsorbed by the porous adsorbent bed, the mole flux gradually decreases along the length direction, and the transient concentration at the tail of the channel remains constant at zero before the time at which the breakthrough point is reached. During the unsteady adsorption process of carbon dioxide, the porous adsorbent bed is saturated starting from the inlet of the channel, and the mole flux along the length direction rises to the value of the inlet flux. On the other hand, the region in which transient concentration remains at zero diminishes gradually with the increasing adsorption time. The region eventually vanishes when the carbon dioxide is reached at the position of the outlet. After that, the concentration at the outlet increases sharply with increase in Fo, as shown in Fig. 8, until the feed concentration is reached, which indicates the adsorption process has been completed.

The LDF mass transfer coefficient $K^{*}$ is a key parameter determined by the mass transfer resistance of microscopic adsorbent particles. When $K^{*}$ is defined to be 9.36 and other parameters is the same as in Table II, the breakthrough curve of the output is calculated as shown in Fig. 10. Compared to the breakthrough curve for $K^{*}=93.6$, the 
appearance time of the breakthrough point is reduced at lower LDF mass transfer coefficient; while, the time until the saturation point is reached is significantly prolonged. This result shows that the LDF mass transfer coefficient plays a considerable role in the flow and adsorption processes. When the mass transfer coefficient rate of the adsorbent particles decreases, the influent carbon dioxide is unable to be adsorbed adequately by the adsorbent bed due to a higher mass transfer resistance; therefore, the residual carbon dioxide flow to the outlet will lead to faster appearance of the breakthrough point than that of the breakthrough curve with the higher LDF mass transfer coefficient. On the other hand, owing to the constant total adsorption capacity of adsorbent bed, the completion time of the adsorption process is extended with the decreasing adsorption efficiency of the adsorbent particles; as a result, the appearance time of the saturation point is prolonged at the higher mass transfer resistance.

\section{CONCLUSION}

In this paper, we modified an MRT-LB model for the CDE to solve the transfer problem with an unsteady source term in porous media at the REV scale, and the correct macroscopic equations can be recovered using the Chapman-Enskog analysis. According to the comparison with the results obtained using the finite-difference method and the analytical solutions, the present MRT-LB model can be applied to calculate the momentum transfer and solve the convection-diffusion problem in porous media. The validated MRT-LB algorithm, which is coupled with the LDF model, was used to analyse the carbon dioxide flow and adsorption behaviours in a porous fixed bed. The breakthrough curve of adsorption from MRT-LB simulation is compared with 
the experimental data and the finite-element solution, and the comparison result shows that this LB model can be used to simulate the flow, mass transfer and adsorption process in porous adsorbent equipment at the REV scale. Then the transient concentration distributions of the carbon dioxide along the porous fixed bed are elaborated in detail. In addition, numerical results show that the LDF mass transfer coefficient plays a considerable role in the flow and adsorption processes. As the mass transfer resistance of the adsorbent particles increases, the appearance time of the breakthrough point is advanced; in contrast, the appearance time of the saturation point is prolonged.

\section{ACKNOWLEDGMENTS}

The authors gratefully acknowledge the supports provided by National Natural Science Foundation of China (No. 51676037), China Scholarship Council (CSC) and the Priority Academic Program Development of Jiangsu Higher Education Institutions. We would also like to thank Dr. Zhenhua Chai, Dr. Wenbin Zhang and Dr. Otobong Archilleus for helpful discussions.

\section{APPENDIX A: CHAPMAN - ENSKOG ANALYSIS ON MRT-LBM FOR CDE}

The Chapman - Enskog analysis is employed to derive the macroscopic equation from the MRT-LB model for CDE. The distribution function, the derivatives of time and space, and the source term can be expanded as:

$$
\begin{gathered}
\mathrm{g}_{i}=\mathrm{g}_{i}^{(0)}+\delta \mathrm{g}_{i}^{(1)}+\delta^{2} \mathrm{~g}_{i}^{(2)}+\ldots, \\
\partial_{t}=\delta \partial_{t 1}+\delta^{2} \partial_{t 2},
\end{gathered}
$$




$$
\begin{gathered}
\partial_{\alpha}=\delta \partial_{\alpha 1}, \\
R=\delta R_{1}, \quad \boldsymbol{F}=\delta \boldsymbol{F}_{1} .
\end{gathered}
$$

Using the Taylor expansion, Eqs. (A1) can be substituted into the evolution equation Eq.(22), thus the evolution equation of zero-,first- and second-order are written as:

$$
\begin{gathered}
\delta^{0}: \mathrm{g}_{i}^{(0)}=\mathrm{g}_{i}^{e q}, \\
\delta^{1}: \quad\left(\partial_{\mathrm{t} 1}+\mathbf{c}_{i} \nabla_{1}\right) \mathrm{g}_{i}^{(0)}=-\frac{1}{\delta t}\left(\mathbf{M}^{-1} \boldsymbol{\Lambda} \mathbf{M}\right)_{i j} \mathrm{~g}_{j}^{(1)}+\left[\mathbf{M}^{-1}\left(\mathbf{I}-\frac{\boldsymbol{\Lambda}}{2}\right) \mathbf{M}\right]_{i j} R_{1, j}, \\
\delta^{2}: \partial_{t_{2}} g_{i}^{(0)}+\left(\partial_{\mathrm{t} 1}+\mathbf{c}_{i} \nabla_{1}\right) \mathrm{g}_{i}^{(1)}+\frac{\delta t}{2}\left(\partial_{\mathrm{t} 1}+\mathbf{c}_{i} \nabla_{1}\right)^{2} \mathrm{~g}_{i}^{(0)}=-\frac{1}{\delta t}\left(\mathbf{M}^{-1} \boldsymbol{\Lambda} \mathbf{M}\right)_{i j} \mathrm{~g}_{j}^{(2)}, \\
\text { where } \quad R_{1, i}=\omega_{\mathrm{i}}\left[\left(1+\frac{\boldsymbol{e}_{i} \cdot \boldsymbol{u}}{\varepsilon c_{s}^{2}}\right) R_{i}+\frac{\boldsymbol{e}_{i} \cdot\left(\varepsilon p \nabla_{1} C / \rho_{0}+C \boldsymbol{F}_{1}\right)}{c_{s}^{2}}\right] .
\end{gathered}
$$

If the distribution functions are rewritten as macroscopic variables in the moment space by multiplying the transformation matrix $\boldsymbol{M}$, Eqs.(A2) in the moment space are modified as:

$$
\begin{gathered}
\delta^{0}: \mathbf{m}^{(0)}=\mathbf{m}^{\mathrm{eq}} \\
\delta^{1}: \boldsymbol{D}_{1} \boldsymbol{m}^{(0)}=-\frac{1}{\delta t} \boldsymbol{\Lambda} \boldsymbol{m}^{(1)}+\left(\boldsymbol{I}-\frac{\boldsymbol{\Lambda}}{2}\right) \boldsymbol{M} \boldsymbol{R}_{1} \\
\delta^{2}: \partial_{t_{2}} \mathbf{m}^{(0)}+\mathbf{D}_{1}\left(\mathbf{I}-\frac{\boldsymbol{\Lambda}}{2}\right) \mathbf{m}^{(1)}+\frac{\delta t}{2} \mathbf{D}_{1}\left(\mathbf{I}-\frac{\boldsymbol{\Lambda}}{2}\right) \mathbf{M R}_{1}=-\frac{1}{\delta t} \boldsymbol{\Lambda} \mathbf{m}^{(2)} \\
\text { where } \quad \boldsymbol{D}_{1}=\partial_{t 1} \boldsymbol{I}+\boldsymbol{M} \partial_{\alpha 1} \operatorname{diag}\left(e_{0, \alpha}, e_{1, \alpha}, \ldots, e_{8, \alpha}\right) \boldsymbol{M}^{-1}
\end{gathered}
$$


be derived as follows [46]:

$$
\begin{gathered}
\sum_{i} \mathrm{~g}_{i}^{(0)}=\sum_{i} \mathrm{~g}_{i}^{\mathrm{eq}}=\sum_{i} \mathrm{~g}_{i}+\frac{\delta t}{2} R=\varepsilon C \\
\sum_{i} \mathrm{~g}_{i}^{(1)}=-\frac{\delta \mathrm{t}}{2} R_{1}
\end{gathered}
$$

Thus, the first term in Eq.(A4b) can be expanded as: 


$$
\partial_{t 1}(\varepsilon C)+\partial_{x 1}\left(C u_{x}\right)+\partial_{y 1}\left(C u_{y}\right)=R_{1}
$$

Analogously, based on Eqs.(24), (A3)and (A6), the fourth, and sixth term in Eq.(A4b) can be calculated as:

$$
\begin{aligned}
& \partial_{t 1}\left(C u_{x}\right)+\partial_{x 1}\left[C\left(\varepsilon c_{s}^{2}+\varepsilon p / \rho_{0}+u_{x}^{2} / \varepsilon\right)\right]+\partial_{y 1}\left(C u_{x} u_{y} / \varepsilon\right) \\
& =-\frac{\lambda_{3}}{\delta t} m_{3}^{(1)}+\left(1-\frac{\lambda_{3}}{2}\right)\left(\varepsilon \frac{p}{\rho_{0}} \partial_{x 1} C+C F_{x 1}+u_{x} \frac{R_{1}}{\varepsilon}\right) \\
& \partial_{t 1}\left(C u_{y}\right)+\partial_{y 1}\left[C\left(\varepsilon c_{s}^{2}+\varepsilon p / \rho_{0}+u_{y}^{2} / \varepsilon\right)\right]+\partial_{x 1}\left(C u_{x} u_{y} / \varepsilon\right) \\
& =-\frac{\lambda_{5}}{\delta t} m_{5}^{(1)}+\left(1-\frac{\lambda_{5}}{2}\right)\left(\varepsilon \frac{p}{\rho_{0}} \partial_{y 1} C+C F_{y 1}+u_{y} \frac{R_{1}}{\varepsilon}\right)
\end{aligned}
$$

It is noted that the following relationship can be derived using Eqs.(9), (12) and (29):

$$
m_{3}^{(1)}=\sum_{i} e_{\mathrm{i}, x} g_{i}^{(1)}, \quad m_{5}^{(1)}=\sum_{i} e_{\mathrm{i}, y} g_{i}^{(1)}
$$

According to Eqs.(A6), (A7) and (A10), the first term of Eq.(A4c) can be derived as:

$$
\begin{aligned}
& \partial_{t 2}(\varepsilon C)+\partial_{x 1}\left\{\left(1-\frac{\lambda_{3}}{2}\right)\left[m_{3}^{(1)}+\frac{\delta t}{2}\left(\varepsilon \frac{p}{\rho_{0}} \partial_{x 1} C+C F_{x 1}+u_{x} \frac{R_{1}}{\varepsilon}\right)\right]\right\} \\
& +\partial_{y 1}\left\{\left(1-\frac{\lambda_{5}}{2}\right)\left[m_{5}^{(1)}+\frac{\delta t}{2}\left(\varepsilon \frac{p}{\rho_{0}} \partial_{y 1} C+C F_{y 1}+u_{y} \frac{R_{1}}{\varepsilon}\right)\right]\right\}=0
\end{aligned} .
$$

It is obvious that Eqs.(A9) can be rewritten as :

$$
\begin{aligned}
& m_{3}^{(1)}+\frac{\delta t}{2}\left(\varepsilon \frac{p}{\rho_{0}} \partial_{x 1} C+C F_{x 1}+u_{x} \frac{R_{1}}{\varepsilon}\right) \\
& =-\frac{\delta \mathrm{t}}{\lambda_{3}}\left[\partial_{t 1}\left(C u_{x}\right)+\partial_{x 1}\left(\varepsilon C c_{s}^{2}+\frac{C \varepsilon p}{\rho_{0}}+\frac{C u_{x}^{2}}{\varepsilon}\right)+\partial_{y 1}\left(\frac{C u_{x} u_{y}}{\varepsilon}\right)-\left(\frac{\varepsilon p}{\rho_{0}} \partial_{x 1} C+C F_{x 1}+u_{x} \frac{R_{1}}{\varepsilon}\right)\right] \\
& =-\frac{\delta \mathrm{t}}{\lambda_{3}}\left[u_{x} \partial_{t 1} C+C\left(\partial_{t 1} u_{x}+\frac{u_{x}}{\varepsilon} \partial_{x 1} u_{x}+\frac{u_{y}}{\varepsilon} \partial_{y 1} u_{x}+\frac{\partial_{x 1} \varepsilon p}{\rho_{0}}\right)+\frac{u_{x}}{\varepsilon}\left(\partial_{x 1} C u_{x}+\partial_{y 1} C u_{y}\right)+\partial_{x 1}\left(\varepsilon C c_{s}^{2}\right)-\left(C F_{x 1}+u_{x} \frac{R_{1}}{\varepsilon}\right)\right]
\end{aligned}
$$

$$
\begin{aligned}
& m_{5}^{(1)}+\frac{\delta t}{2}\left(\varepsilon \frac{p}{\rho_{0}} \partial_{y 1} C+C F_{y 1}+u_{y} \frac{R_{1}}{\varepsilon}\right) \\
& =-\frac{\delta \mathrm{t}}{\lambda_{5}}\left[\partial_{t 1}\left(C u_{y}\right)+\partial_{y 1}\left(\varepsilon C c_{s}^{2}+\frac{C \varepsilon p}{\rho_{0}}+\frac{C u_{y}^{2}}{\varepsilon}\right)+\partial_{x 1}\left(\frac{C u_{x} u_{y}}{\varepsilon}\right)-\left(\frac{\varepsilon p}{\rho_{0}} \partial_{y 1} C+C F_{y 1}+u_{y} \frac{R_{1}}{\varepsilon}\right)\right] \\
& =-\frac{\delta \mathrm{t}}{\lambda_{5}}\left[u_{y} \partial_{t 1} C+C\left(\partial_{t 1} u_{y}+\frac{u_{y}}{\varepsilon} \partial_{y 1} u_{y}+\frac{u_{x}}{\varepsilon} \partial_{x 1} u_{y}+\frac{\partial_{y 1} \varepsilon p}{\rho_{0}}\right)+\frac{u_{y}}{\varepsilon}\left(\partial_{y 1} C u_{y}+\partial_{x 1} C u_{x}\right)+\partial_{y 1}\left(\varepsilon C c_{s}^{2}\right)-\left(C F_{y 1}+u_{y} \frac{R_{1}}{\varepsilon}\right)\right]
\end{aligned}
$$


If Eq.(A8) is substituted into the right -side of Eqs.(A12) and (A13), we can get that:

$$
\begin{aligned}
m_{3}^{(1)}+\frac{\delta t}{2}\left(\varepsilon \frac{p}{\rho_{0}} \partial_{x 1} C+C F_{x 1}+u_{x} \frac{R_{1}}{\varepsilon}\right) \\
=-\frac{\delta \mathrm{t}}{\lambda_{3}}\left[C\left(\partial_{t 1} u_{x}+\frac{u_{x}}{\varepsilon} \partial_{x 1} u_{x}+\frac{u_{y}}{\varepsilon} \partial_{y 1} u_{x}+\frac{\partial_{x 1} \varepsilon p}{\rho_{0}}-F_{x 1}\right)+\partial_{x 1}\left(\varepsilon C c_{s}^{2}\right)\right] \\
m_{5}^{(1)}+\frac{\delta t}{2}\left(\varepsilon \frac{p}{\rho_{0}} \partial_{y 1} C+C F_{y 1}+u_{y} \frac{R_{1}}{\varepsilon}\right) \\
=-\frac{\delta \mathrm{t}}{\lambda_{5}}\left[C\left(\partial_{t 1} u_{y}+\frac{u_{x}}{\varepsilon} \partial_{x 1} u_{y}+\frac{u_{y}}{\varepsilon} \partial_{y 1} u_{y}+\frac{\partial_{y 1} \varepsilon p}{\rho_{0}}-F_{y 1}\right)+\partial_{y 1}\left(\varepsilon C c_{s}^{2}\right)\right]
\end{aligned}
$$

On the other hand, based on Champan-Enskog analysis of the LBM momentum model in porous media, the first-order Navier-Stokes equation is expressed as:

$$
\partial_{t 1} \boldsymbol{u}+\left(\boldsymbol{u} \cdot \nabla_{1}\right)\left(\frac{\boldsymbol{u}}{\varepsilon}\right)=-\frac{1}{\rho_{0}} \nabla_{1}(\varepsilon p)+\boldsymbol{F}_{1} .
$$

Comparing Eq.(A16) with the right-side of Eqs.(A14) and (A15), it is easy to obtain that:

$$
\begin{aligned}
& m_{3}^{(1)}+\frac{\delta t}{2}\left(\varepsilon \frac{p}{\rho_{0}} \partial_{x 1} C+C F_{x 1}+u_{x} \frac{R_{1}}{\varepsilon}\right)=-\frac{\delta \mathrm{t}}{\lambda_{3}} \partial_{x 1}\left(\varepsilon C c_{s}^{2}\right) \\
& m_{5}^{(1)}+\frac{\delta t}{2}\left(\varepsilon \frac{p}{\rho_{0}} \partial_{y 1} C+C F_{y 1}+u_{y} \frac{R_{1}}{\varepsilon}\right)=-\frac{\delta \mathrm{t}}{\lambda_{5}} \partial_{y 1}\left(\varepsilon C c_{s}^{2}\right)
\end{aligned}
$$

Therefore , according to Eqs.(A17) and (A18), Eq.(A11) can be rewritten as:

$$
\partial_{t 2}(\varepsilon C)+\partial_{x 1}\left[\left(\frac{1}{2}-\frac{1}{\lambda_{3}}\right) \varepsilon c_{s}^{2} \partial_{x 1} C\right]+\partial_{y 1}\left[\left(\frac{1}{2}-\frac{1}{\lambda_{5}}\right) \varepsilon c_{s}^{2} \partial_{y 1} C\right]=0
$$

Utilizing Eqs.(A8) and (A19), the macroscopic CDE can be obtained:

$$
\varepsilon \frac{\partial C}{\partial t}+\nabla(\mathbf{u} C)=\varepsilon D \nabla^{2} C+R
$$

where the diffusion coefficient $D$ is given by:

$$
D=c_{s}^{2}\left(\frac{1}{\lambda_{3}}-\frac{1}{2}\right) \delta t=c_{s}^{2}\left(\frac{1}{\lambda_{5}}-\frac{1}{2}\right) \delta t
$$

In general, the source term $R$ in Eq.(A20) represents an universal source/sink term 
in CDE. For mass transfer and adsorption processes in porous media, the source term $R$ is used to describe the adsorption behaviors caused by the adsorbent particles, which is determined by LDF model:

$$
R=R^{*}=(1-\varepsilon) \rho_{b} K_{L}\left(q^{*}-\bar{q}\right)
$$

It is obvious that the developed LB model will be recovered as Eq.(3).

\section{APPENDIX B: ANALYTICAL SOLUTIONS OF THERMAL POISEUILLE FLOW}

Based on the research results form the literature [37], the analytical solutions of velocity and temperature along the y-axis, and the heat flux $\left(J_{x}, J_{y}\right)$ can be obtained as:

$$
\begin{gathered}
\boldsymbol{u}_{y}=4 U_{\max } \frac{y}{H}\left(1-\frac{y}{H}\right), \\
\frac{T-T_{1}}{T_{0}-T_{1}}=\frac{y}{H}+\frac{\operatorname{Pr} E c}{3}\left[1-\left(1-\frac{2 y}{H}\right)^{4}\right], \\
J_{x}=4 U_{\max } \frac{y}{H}\left(1-\frac{y}{H}\right) T, \\
J_{\mathrm{y}}=-D \frac{\left(T_{0}-T_{1}\right)}{H}\left[1+\frac{8 \operatorname{Pr} E c}{3}\left(1-\frac{2 y}{H}\right)^{3}\right],
\end{gathered}
$$

\section{APPENDIX C: ANALYTICAL SOLUTION OF UNSTEADY MASS DIFFUSION}

If the fluid flow is not considered, the CDE as Eq.(3) will be converted into a unsteady diffusion equation with a source term. Using the method of variable separation, the transient concentration along $y$-axis can be expressed as:

$$
C(\tau, y)=\sum_{n=1}^{\infty} \Theta_{n}(\tau) \sin \frac{n \pi}{H} y
$$




$$
\begin{gathered}
\text { where } \Theta_{n}(\tau)=\int_{0}^{\tau} \psi_{n} \cdot e^{-D\left[\left(\frac{n \pi}{H}\right)^{2}+\frac{k_{m}}{\varepsilon}\right](\tau-t)} d t \\
\text { and } \psi_{n}=\frac{2}{H} \int_{0}^{H} \frac{k_{m}}{\varepsilon} C_{1} \sin \left(\frac{n \pi}{H} y\right) d y,
\end{gathered}
$$

where the computation time $\tau$ can be integrated into the dimensionless time Fo number.

\section{References}

[1] N. Sun, C. Sun, H. Liu, J. Liu, L. Stevens , T. Drage, C. E. Snape, K. Li, W. Wei, and Y. Sun, Fuel, 113, 854(2013).

[2] F. Liu, X. Yan, F. Fan, C. Zhao, R. Liu, Y.Gao, and Y. Wang, Micro Nano Lett. 11, 372(2016).

[3] N. Manjhi, N. Verma, K. Salem, and D. Mewes, Chem. Eng. Sci. 61, 7754 (2006).

[4] M. Gholami, M. R. Talaie, and Roodpeyma, S. Chem. Eng. Sci. 65, 5942 (2010).

[5] K. S. Hwang, J. H. Jun, and W. K. Lee, Chem. Eng. Sci. 50, 813(1995).

[6] R. T. Yang, Gas Separation by Adsorption Processes.(Butterworth, Boston,1987).

[7] L. Fournel, P. Mocho, R. Brown, and P. Le Cloirec, Adsorption 16, 147(2010).

[8] L. Hauchhum, P. Mahanta, J. De Wilde, Transp. Porous Med. 110, 503(2015).

[9] M. S. Shafeeyan, W. M. A. W. Daud, and A. Shamiri, Chem. Eng. Res. Des. 92, 961(2014).

[10]Q. Li, K. H. Luo, Q. J. Kang, Y. L. He, Q. Chen,, and Q. Liu, Prog. Energ. Combust. 52, 62(2016).

[11]B.H. Wen, C. Y. Zhang, and H. P. Fang, Entropy 17, 8240(2015) .

[12]Q. Ma, and Z.Q. Chen, Phys. Rev. E, 92, 013025(2015).

[13]Q. Liu, and Y. L. He, Phys. A 429, 215(2015).

[14]J.Wang, D.Wang, P. Lallemand, and L.-S. Luo, Comput. Math. Appl. 65, 262 (2013).

[15]I. Rasin, S. Succi, and W. Miller, J. Comput. Phys. 206, 45(2005).

[16]I. Ginzburg, Commun. Comput. Phys. 11, 1439 (2012).

[17]D. Sun, M. Zhu, J. Wang, and B. Sun, Int. J. Heat Mass Tran. 94, 474 (2016).

[18]Z. Chai, C. Huang, B. Shi, and Z. Guo, Int. J. Heat Mass Tran. 98, 687(2016).

[19]H. Karani, and C. Huber, Phys. Rev. E 91, 023304(2015).

[20]Y. Chen , Z. Deng, J. Fluid Mech. 819, 401 (2017).

[21]M. Levesque, M. Duvail, I. Pagonabarraga, D. Frenkel, and B. Rotenberg, Phys. Rev. E 88, 013308(2013).

[22] Y. Ning, Y. Jiang, H. Liu, and G. Qin, J. Nat. Gas. Sci. Eng. 26, 345(2015).

[23]S. Agarwal, N.Verma, and D. Mewes, Heat Mass Trans. 41, 843 (2005).

[24]N. Manjhi, N. Verma, K. Salem, and D. Mewes, Chem. Eng. Sci. 61, 2510 (2006).

[25]N. Verma, K. Salem, and D. Mewes, Chem. Eng. Sci. 62, 3685(2007).

[26]N. Verma, and D. Mewes, Chem. Eng. Sci. 63, 4269 (2008).

[27]L. Zhou, Z. G. Qu, L. Chen, and W. Q. Tao, J. Comput. Phys. 300, 800(2015).

[28]L. Zhou, Z. G. Qu, T. Ding, and J. Y. Miao, Phys. Rev. E 93, 043101 (2016).

[29]D. Gao , Z. Chen , L. Chen, Int. J. Heat Mass Tran. 70, 979(2014). 
[30]D. Zhang, R. Zhang, S. Chen, and W. E. Soll, Geophys. Res. Lett. 27, 1195(2000).

[31]Z. Guo, and T. S. Zhao, Phys. Rev. E 66,036304(2002).

[32]T. Seta, E.Takegoshi , K. Okui. Math Comput. Simulat. 72, 195(2006).

[33]S. D. C. Walsh, H. Burwinkle, M.O. Saar. Comput. Geosci. 35,1186(2009).

[34]S. Anwar, M. C. Sukop, Comput. Math. Appl., 58, 1015(2009).

[35]Z. Deng, Y. Chen, C. Shao, Phys. Rev. E 93, 013128(2016).

[36]Q. Liu, Y. L. He, Q. Li and W. Q. Tao, Int. J. Heat Mass Tran. 73, 761(2014).

[37]Z. Chai, and T. S. Zhao, Phys. Rev. E 90, 013305(2014).

[38]Z. Chai, and T. S. Zhao, Phys. Rev. E 87, 063309 (2013).

[39]J. Gleuckauf, and J. I. Coates, J. Chem. Soc. 1315, e21.(1947).

[40] S.Sircar, and J. R. Hufton, Adsorption, 6, 137 (2000).

[41]A. Patton, B. D. Crittenden, and S. P. Perera, Chem. Eng. Res. Des. 82, 999(2004).

[42]J. Álvarez-Ramírez, G. Fernández-Anaya, F. J. Valdés-Parada, and J. A. OchoaTapia, Ind. Eng. Chem. Res. 44, 6776(2005).

[43]R. Chauveau, G. Grévillot, S. Marsteau, and C. Vallières, Chem. Eng. Res. Des. 91, 955(2013).

[44]T. L. P. Dantas, F. M. T. Luna, I. J. Silva, D. C. de Azevedo, C. A. Grande, A. E. Rodrigues, and R. F. Moreira, Chem. Eng. J. 169, 11(2011).

[45]R. Du, B. Shi, and X. Chen, Phys. Lett. A 359, 564(2006).

[46]L.Wang, J. Mi, and Z. Guo, Int. J. Heat Mass Tran. 94, 269(2016).

[47]Z.L. Guo, C.G. Zheng, B.C. Shi,Chin. Phys. 11366 (2002).

[48]T. L. P. Dantas, S. M.Amorim, F. M. T. Luna, I. J. Silva Jr, D. C. de Azevedo,

A. E.Rodrigues, and R. F. Moreira, Sep. Sci. Technol. 45 73(2010).

[49]J. Toth, Acta Chem. Acad. Hung. 69, 311(1971).

Table captions:

Table I Parameters of Toth model

Table II Physical and transport properties of fluid and porous fixed bed

\section{Figure captions:}

FIG.1 Schematic of relationship between the pore scale and the REV scale

FIG.2 Schematic of the Poiseuille flow in a 2D porous channel.

FIG.3 Velocity profiles of the Poiseuille flow for different Reynolds and Darcy numbers.

(a) $D a=10^{-5}$, (b) $R e=0.1$.

FIG.4 Comparisons between MRT-LB results and analytical solutions of the thermal

Poiseuille flow.(a) Temperature, (b)Flux $J_{x}^{*}$, (c)Flux $J_{y}^{*}$.

FIG.5 Comparisons between MRT-LB results and FDM solutions of the thermal Poiseuille flow in 2D porous channel.(a) Temperature, (b)Flux $J_{x}^{*}$.

FIG.6 Comparisons between MRT-LB results and analytical solutions of the mass 
diffusion with an unsteady state source term..(a) $k_{m}^{*}=0.72$, (b) $k_{m}^{*}=7.2$, (c) $k_{m}^{*}=36$.

FIG.7 Schematic of the transfer and adsorption of carbon dioxide in a porous fixed bed. FIG.8 Breakthrough curves of adsorption process.

FIG.9 Numerical results of the mole flux distribution and transient concentration along length direction.(a) mole flux, (b) transient concentration.

FIG.10 Effect of LDF mass transfer coefficient on breakthrough curves.

Table I Parameters of Toth model

\begin{tabular}{ccc}
\hline \hline Parameters & Units & Value \\
\hline$q_{m}$ & $\mathrm{~mol} / \mathrm{kg}$ & 10.05 \\
$\varphi$ & $/$ & 0.678 \\
$-\Delta H$ & $\mathrm{~kJ} / \mathrm{mol}$ & 21.84 \\
$K_{e q}^{0}$ & $\mathrm{bar}^{-1}$ & 7.62 \\
\hline
\end{tabular}


Table II Physical and transport properties of fluid and porous fixed bed

\begin{tabular}{cc}
\hline \hline $\begin{array}{c}\text { Dimensionless } \\
\text { numbers }\end{array}$ & Value \\
\hline$\varepsilon$ & 0.52 \\
$R e$ & 9 \\
$S c$ & 2.51 \\
$K^{*}$ & 93.6 \\
$D a$ & $2 \times 10^{-6}$ \\
\hline
\end{tabular}






Pore scale

REV scale

FIG.1 Schematic of the relationship between the pore scale and the REV scale. 




Wall

FIG.2 Schematic of the Poiseuille flow in a 2D porous channel. 




(a)



(b) 
FIG.3 Velocity profiles of the Poiseuille flow for different Reynolds and Darcy numbers. (a) $D a=10^{-5}$, (b) $R e=0.1$.

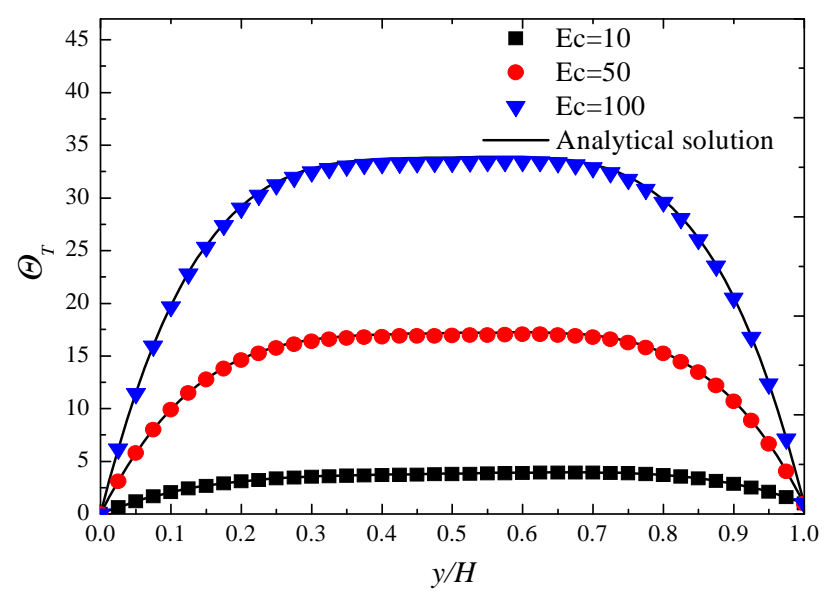

(a)

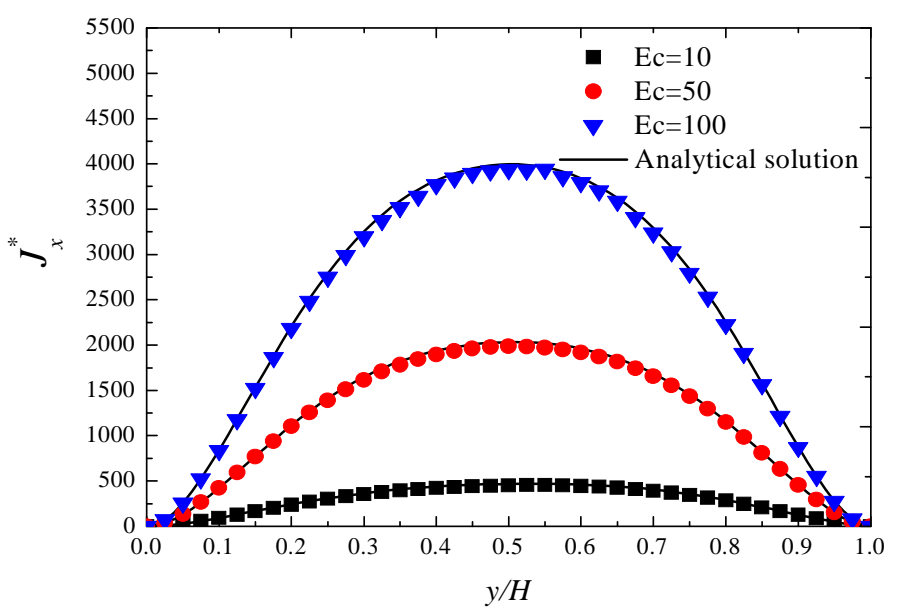

(b)

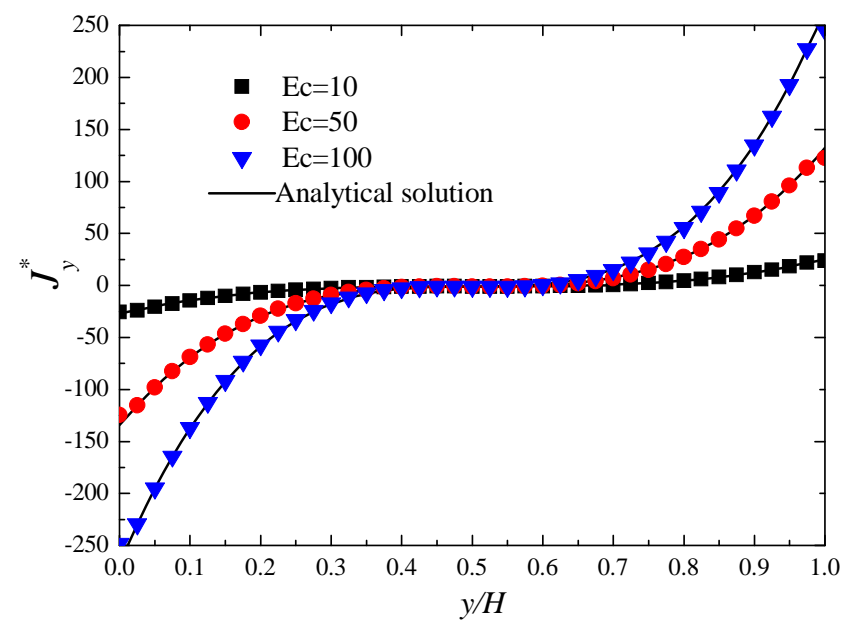


(c)

FIG.4 Comparisons between MRT-LB results and analytical solutions of the thermal

Poiseuille flow.(a) Temperature, (b)Flux $J_{x}^{*}$, (c)Flux $J_{y}^{*}$.



(a)

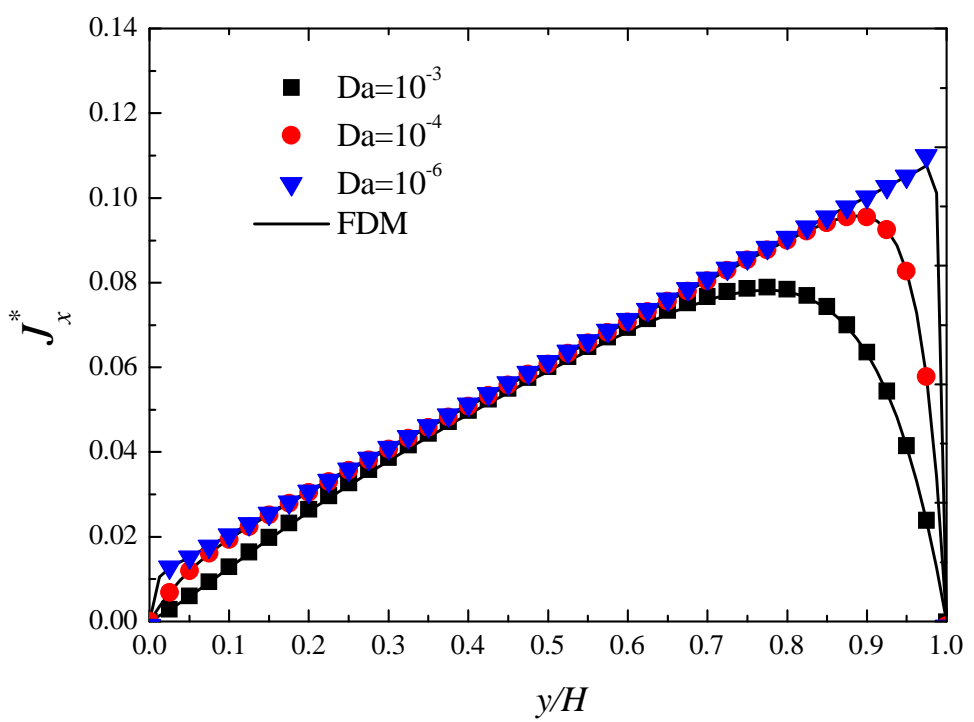

(b)

FIG.5 Comparisons between MRT-LB results and FDM solutions of the thermal 
Poiseuille flow in 2D porous channel.(a) Temperature, (b)Flux $J_{x}^{*}$.

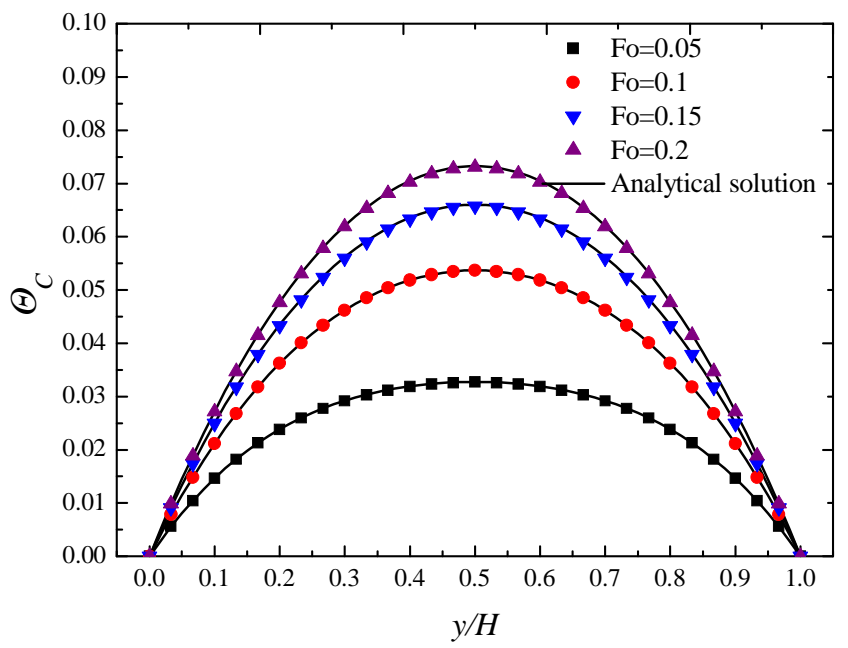

(a)

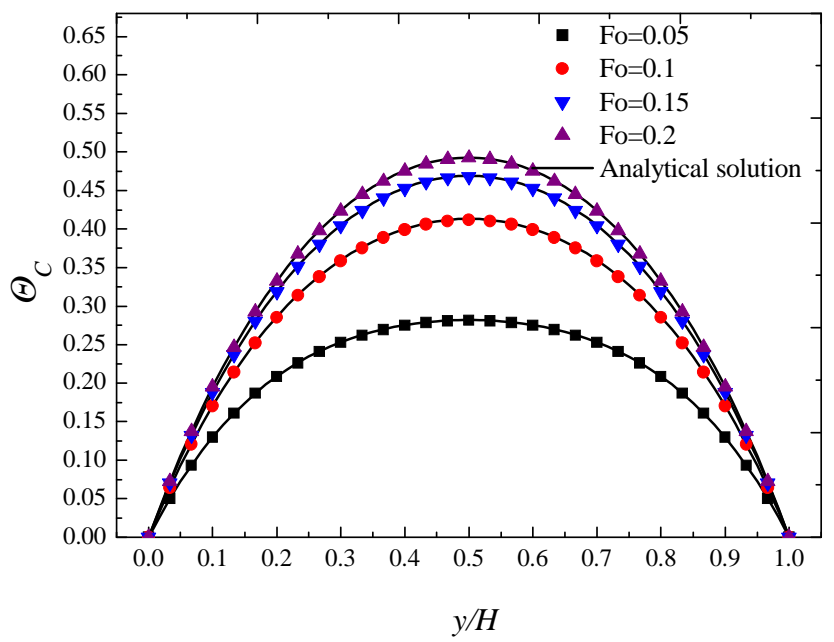

(b) 


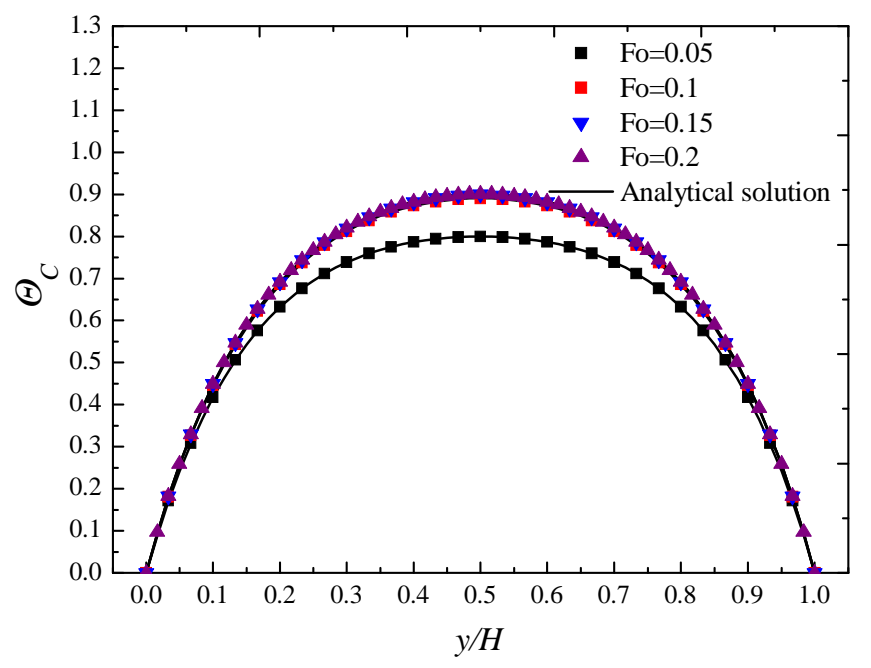

(c)

FIG.6 Comparisons between MRT-LB results and analytical solutions of the mass diffusion with an unsteady state source term.(a) $k_{m}^{*}=0.72$, (b) $k_{m}^{*}=7.2$, (c) $k_{m}^{*}=36$.



FIG.7 Schematic of the transfer and adsorption of carbon dioxide in a porous

fixed bed 


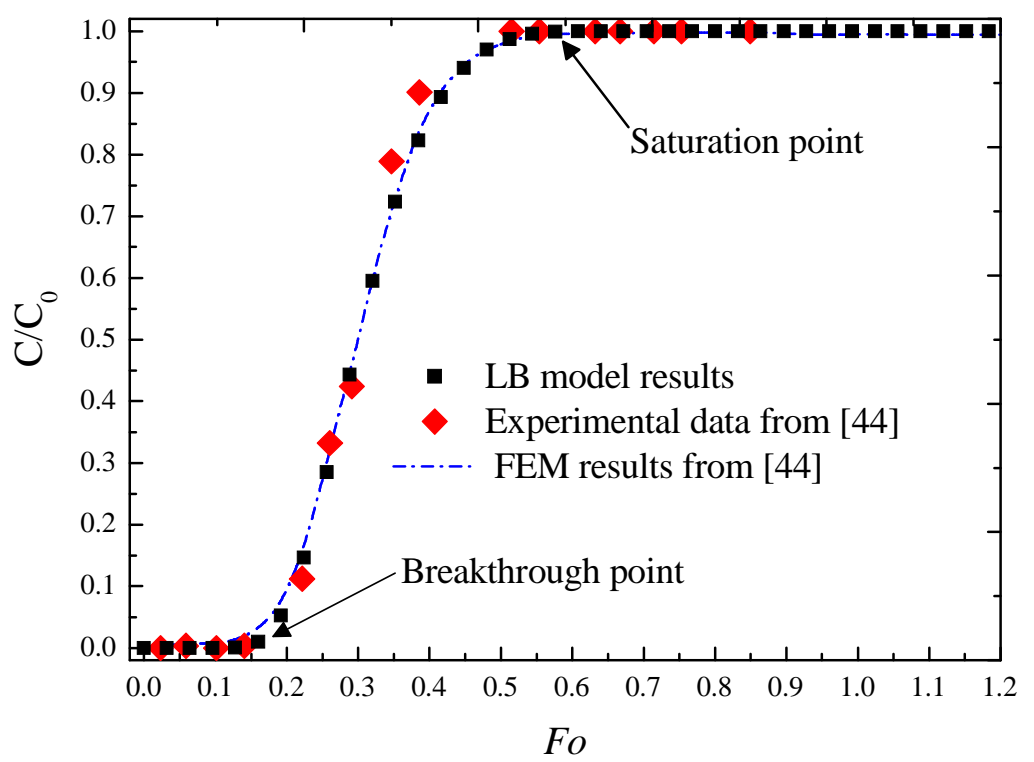

FIG.8 Breakthrough curves of adsorption process. 


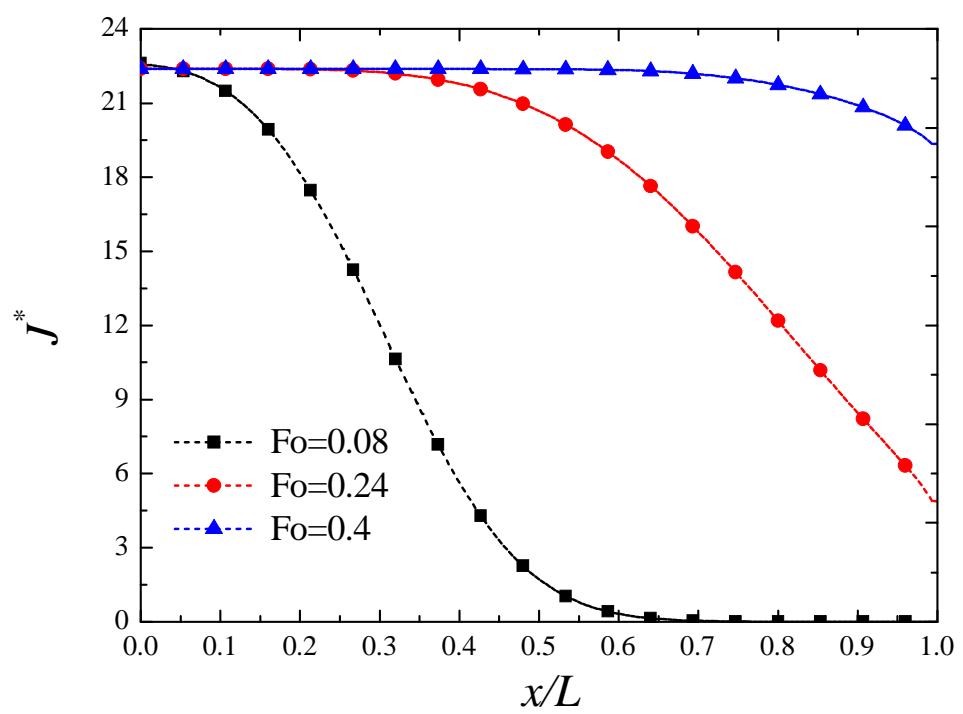

(a)

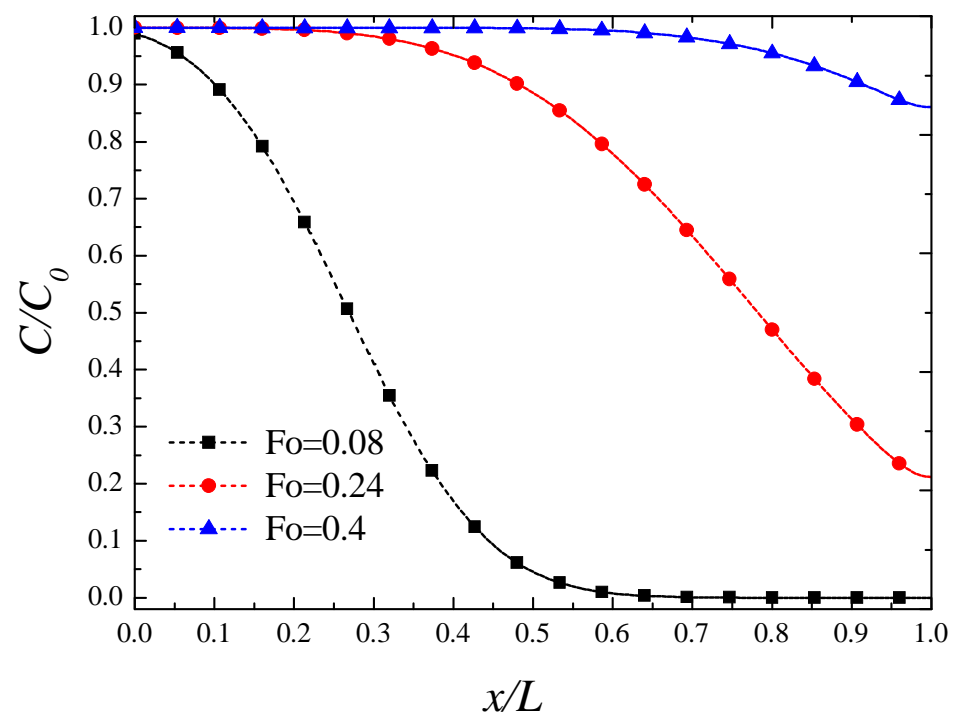

(b)

FIG.9 Numerical results of the mole flux distribution and transient concentration along length direction.(a) mole flux, (b) transient concentration. 


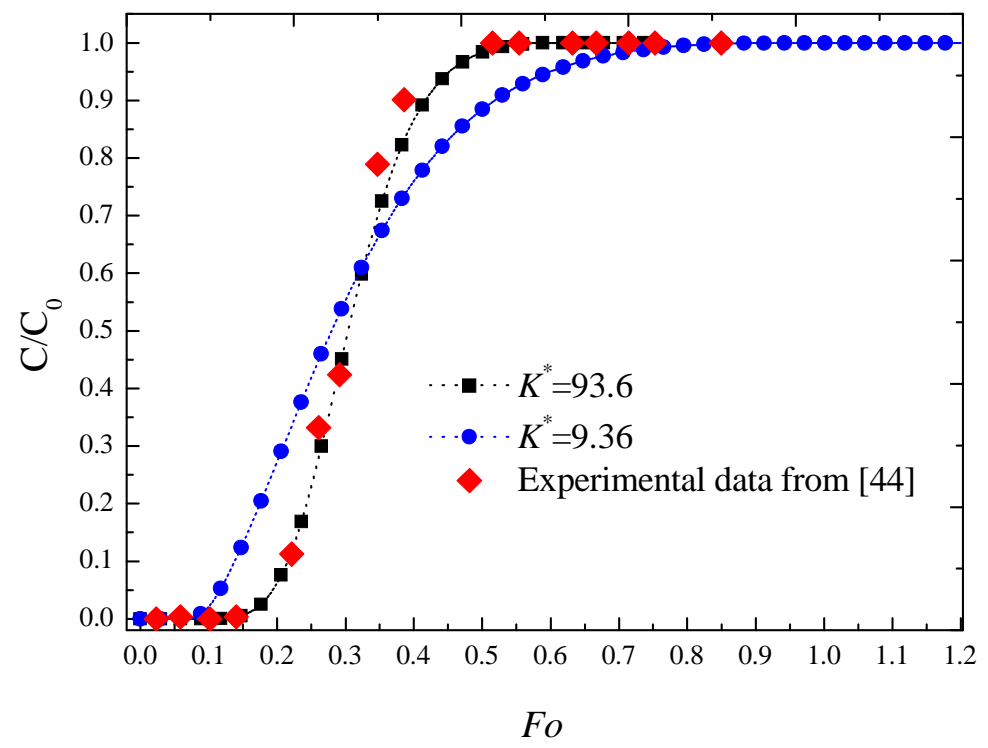

FIG.10 Effect of LDF mass transfer coefficient on breakthrough curves. 